Supplement of

\title{
Physical and optical properties of aged biomass burning aerosol from wildfires in Siberia and the Western USA at the Mt. Bachelor Observatory
}

James R. Laing et al.

Correspondence to: Daniel A. Jaffe (djaffe@uw.edu)

The copyright of individual parts of the supplement might differ from the CC-BY 3.0 licence. 
Table S1. Optical properties of the identified BB plumes at MBO during the summer of 2015. All enhancement ratios are obtained by taking the slope of a RMA linear regression between the two species. Precision uncertainty and total uncertainty are provided for each value. ND ("no data") indicates missing data. WC in the MAE column signifies a weak correlation $\left(\mathrm{R}^{2}<0.60\right)$.

\begin{tabular}{|c|c|c|c|c|c|c|c|c|}
\hline $\begin{array}{c}\text { Event } \\
\text { number }\end{array}$ & Event date and time (UTC) & & $\begin{array}{c}\Delta \sigma_{\text {scat }} / \Delta C O \\
\left(\mathrm{Mm}^{-1} \mathrm{ppbv}^{-1}\right)\end{array}$ & $\begin{array}{c}\Delta \sigma_{\mathrm{abs}} / \Delta \mathrm{CO} \\
\left(\mathrm{Mm}^{-1} \mathrm{ppbv}^{-1}\right)\end{array}$ & $\begin{array}{c}\text { MSE } \\
\left(m^{2} g^{-1}\right)\end{array}$ & $\begin{array}{c}\text { MAE } \\
\left(m^{2} g^{-1}\right)\end{array}$ & $\begin{array}{c}\text { AAE } \\
(467-660 \mathrm{~nm})\end{array}$ & $\begin{array}{c}\omega \\
(528 \mathrm{~nm}) \\
\end{array}$ \\
\hline \multirow{3}{*}{1} & \multirow{3}{*}{ 7/31/15 15:35-17:10 } & mean & 1.13 & 0.036 & ND & ND & 3.15 & 0.97 \\
\hline & & precision uncertainty & 0.17 & 0.003 & ND & ND & 0.29 & 0.15 \\
\hline & & total uncertainty & 0.19 & 0.012 & ND & ND & 1.32 & 0.35 \\
\hline \multirow{3}{*}{2} & \multirow{3}{*}{ 8/9/15 2:55-8:55 } & mean & 0.89 & WC & 3.17 & 0.085 & 3.45 & 0.98 \\
\hline & & precision uncertainty & 0.13 & WC & 0.46 & 0.011 & 0.33 & 0.15 \\
\hline & & total uncertainty & 0.14 & WC & 0.51 & 0.034 & 1.60 & 0.41 \\
\hline \multirow{3}{*}{3} & \multirow{3}{*}{$8 / 9 / 15 \quad 13: 35-8 / 10 / 15 \quad 0: 00$} & mean & 1.24 & 0.033 & 3.29 & 0.087 & 3.72 & 0.98 \\
\hline & & precision uncertainty & 0.18 & 0.002 & 0.47 & 0.009 & 0.35 & 0.15 \\
\hline & & total uncertainty & 0.20 & 0.012 & 0.52 & 0.032 & 1.68 & 0.38 \\
\hline \multirow{3}{*}{4} & \multirow{3}{*}{$8 / 10 / 15 \quad 1: 10-5: 55$} & mean & 1.05 & 0.030 & 3.78 & 0.108 & 3.86 & 0.97 \\
\hline & & precision uncertainty & 0.15 & 0.002 & 0.54 & 0.011 & 0.34 & 0.15 \\
\hline & & total uncertainty & 0.17 & 0.010 & 0.61 & 0.036 & 1.62 & 0.35 \\
\hline \multirow{3}{*}{5} & \multirow{3}{*}{$8 / 10 / 15 \quad 6: 05-11: 40$} & mean & 1.09 & 0.034 & 3.44 & 0.106 & 4.02 & 0.97 \\
\hline & & precision uncertainty & 0.15 & 0.003 & 0.49 & 0.011 & 0.36 & 0.15 \\
\hline & & total uncertainty & 0.17 & 0.012 & 0.55 & 0.037 & 1.78 & 0.36 \\
\hline \multirow{3}{*}{6} & \multirow{3}{*}{ 8/10/15 11:45-14:35 } & mean & 0.94 & WC & 3.27 & WC & 4.12 & 0.99 \\
\hline & & precision uncertainty & 0.13 & WC & 0.48 & WC & 0.38 & 0.15 \\
\hline & & total uncertainty & 0.15 & WC & 0.53 & WC & 1.89 & 0.38 \\
\hline \multirow{3}{*}{7} & \multirow{3}{*}{$8 / 10 / 15 \quad 14: 40-8 / 11 / 15 \quad 6: 15$} & mean & 1.17 & 0.032 & 3.64 & 0.098 & 3.52 & 0.98 \\
\hline & & precision uncertainty & 0.17 & 0.002 & 0.52 & 0.010 & 0.31 & 0.15 \\
\hline & & total uncertainty & 0.19 & 0.011 & 0.58 & 0.035 & 1.55 & 0.37 \\
\hline \multirow{3}{*}{8} & \multirow{3}{*}{$8 / 11 / 15 \quad 14: 20-18: 45$} & mean & 1.07 & 0.029 & 2.5 & 0.066 & 2.74 & 0.98 \\
\hline & & precision uncertainty & 0.16 & 0.003 & 0.36 & 0.008 & 0.28 & 0.15 \\
\hline & & total uncertainty & 0.18 & 0.010 & 0.40 & 0.023 & 1.14 & 0.36 \\
\hline \multirow{3}{*}{9} & \multirow{3}{*}{ 8/14/15 10:00-15:35 } & mean & 0.48 & 0.007 & 2.75 & 0.042 & 3.06 & 0.99 \\
\hline & & precision uncertainty & 0.07 & 0.001 & 0.39 & 0.005 & 0.29 & 0.15 \\
\hline & & total uncertainty & 0.08 & 0.003 & 0.44 & 0.018 & 1.44 & 0.43 \\
\hline \multirow{3}{*}{10} & \multirow{3}{*}{$8 / 17 / 15 \quad 0: 05-3: 55$} & mean & 1.39 & 0.078 & ND & ND & 2.48 & 0.95 \\
\hline & & precision uncertainty & 0.20 & 0.006 & ND & ND & 0.21 & 0.14 \\
\hline & & total uncertainty & 0.22 & 0.021 & ND & ND & 0.90 & 0.30 \\
\hline \multirow{3}{*}{11} & \multirow{3}{*}{$8 / 17 / 15 \quad 17: 15-8 / 18 / 15 \quad 7: 00$} & mean & 1.06 & 0.06 & ND & ND & 2.50 & 0.95 \\
\hline & & precision uncertainty & 0.15 & 0.005 & ND & ND & 0.22 & 0.15 \\
\hline & & total uncertainty & 0.17 & 0.020 & ND & ND & 0.92 & 0.30 \\
\hline
\end{tabular}


Table S1. Continued.

\begin{tabular}{|c|c|c|c|c|c|c|c|c|}
\hline $\begin{array}{c}\text { Event } \\
\text { number }\end{array}$ & Event date and time (UTC) & & $\begin{array}{c}\Delta \sigma_{\text {scat }} / \Delta C O \\
\left(\mathrm{Mm}^{-1} \mathrm{ppbv}^{-1}\right)\end{array}$ & $\begin{array}{c}\Delta \sigma_{\mathrm{abs}} / \Delta \mathrm{CO} \\
\left(\mathrm{Mm}^{-1} \mathrm{ppbv}^{-1}\right)\end{array}$ & $\begin{array}{c}\text { MSE } \\
\left(\mathbf{m}^{2} \mathbf{g}^{-1}\right)\end{array}$ & $\begin{array}{c}\text { MAE } \\
\left(\mathrm{m}^{2} \mathrm{~g}^{-1}\right)\end{array}$ & $\begin{array}{c}\text { AAE } \\
(467-660 \mathrm{~nm})\end{array}$ & $\begin{array}{c}\omega \\
(528 \mathrm{~nm})\end{array}$ \\
\hline \multirow{3}{*}{12} & \multirow{3}{*}{ 8/18/15 16:05 - 8/19/15 16:40 } & mean & 1.29 & 0.075 & ND & ND & 2.30 & 0.95 \\
\hline & & precision uncertainty & 0.18 & 0.005 & ND & ND & 0.20 & 0.15 \\
\hline & & total uncertainty & 0.21 & 0.020 & ND & ND & 0.83 & 0.30 \\
\hline \multirow{3}{*}{13} & \multirow{3}{*}{$8 / 19 / 15 \quad 17: 40-8 / 20 / 15 \quad 3: 05$} & mean & 1.12 & 0.052 & ND & ND & 2.25 & 0.96 \\
\hline & & precision uncertainty & 0.16 & 0.004 & ND & ND & 0.20 & 0.15 \\
\hline & & total uncertainty & 0.18 & 0.014 & ND & ND & 0.81 & 0.30 \\
\hline \multirow{3}{*}{14} & \multirow{3}{*}{ 8/22/15 15:30-18:05 } & mean & 1.97 & 0.078 & 4.76 & 0.188 & 3.59 & 0.96 \\
\hline & & precision uncertainty & 0.28 & 0.015 & 0.70 & 0.021 & 0.31 & 0.15 \\
\hline & & total uncertainty & 0.32 & 0.023 & 0.78 & 0.057 & 1.39 & 0.32 \\
\hline \multirow{3}{*}{15} & \multirow{3}{*}{$8 / 23 / 15 \quad 3: 55-7: 00$} & mean & 1.09 & 0.059 & 2.84 & 0.156 & 2.51 & 0.96 \\
\hline & & precision uncertainty & 0.17 & 0.006 & 0.40 & 0.019 & 0.23 & 0.15 \\
\hline & & total uncertainty & 0.18 & 0.019 & 0.46 & 0.050 & 0.98 & 0.33 \\
\hline \multirow{3}{*}{16} & \multirow{3}{*}{$8 / 23 / 15 \quad 9: 50-8 / 25 / 15 \quad 6: 50$} & mean & 1.13 & 0.029 & 4.06 & 0.107 & 3.15 & 0.98 \\
\hline & & precision uncertainty & 0.16 & 0.002 & 0.58 & 0.011 & 0.27 & 0.15 \\
\hline & & total uncertainty & 0.18 & 0.011 & 0.65 & 0.039 & 1.40 & 0.38 \\
\hline \multirow{3}{*}{17} & \multirow{3}{*}{ 8/25/15 $12: 45-8 / 26 / 15 \quad 19: 00$} & mean & 0.88 & 0.027 & 3.75 & 0.111 & 3.12 & 0.98 \\
\hline & & precision uncertainty & 0.12 & 0.002 & 0.53 & 0.012 & 0.27 & 0.15 \\
\hline & & total uncertainty & 0.14 & 0.009 & 0.60 & 0.036 & 1.26 & 0.34 \\
\hline \multirow{3}{*}{18} & \multirow{3}{*}{$8 / 26 / 15 \quad 7: 15-8 / 28 / 15 \quad 11: 15$} & mean & 0.89 & 0.031 & 3.70 & 0.128 & 3.48 & 0.97 \\
\hline & & precision uncertainty & 0.13 & 0.002 & 0.52 & 0.013 & 0.31 & 0.15 \\
\hline & & total uncertainty & 0.14 & 0.010 & 0.59 & 0.043 & 1.46 & 0.35 \\
\hline \multirow{3}{*}{19} & \multirow{3}{*}{$8 / 28 / 15 \quad 17: 40-8 / 29 / 15 \quad 6: 15$} & mean & 0.69 & ND & 2.94 & ND & ND & ND \\
\hline & & precision uncertainty & 0.10 & ND & 0.42 & ND & ND & ND \\
\hline & & total uncertainty & 0.11 & ND & 0.47 & ND & ND & ND \\
\hline
\end{tabular}


Figures:

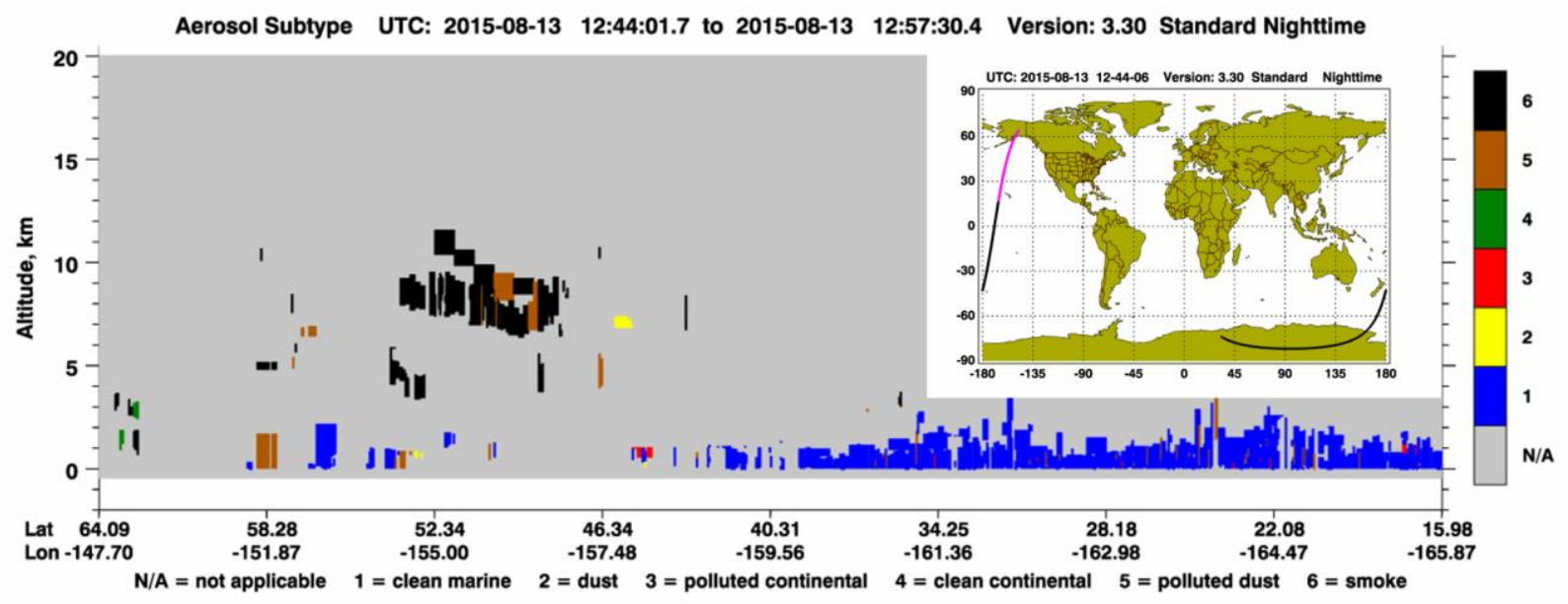

Figure S1. Aerosol types classified by CALIPSO from a transect over the Pacific Ocean on 8/13/2015 12:44 UTC showing a large smoke plume from the Siberian fires. 


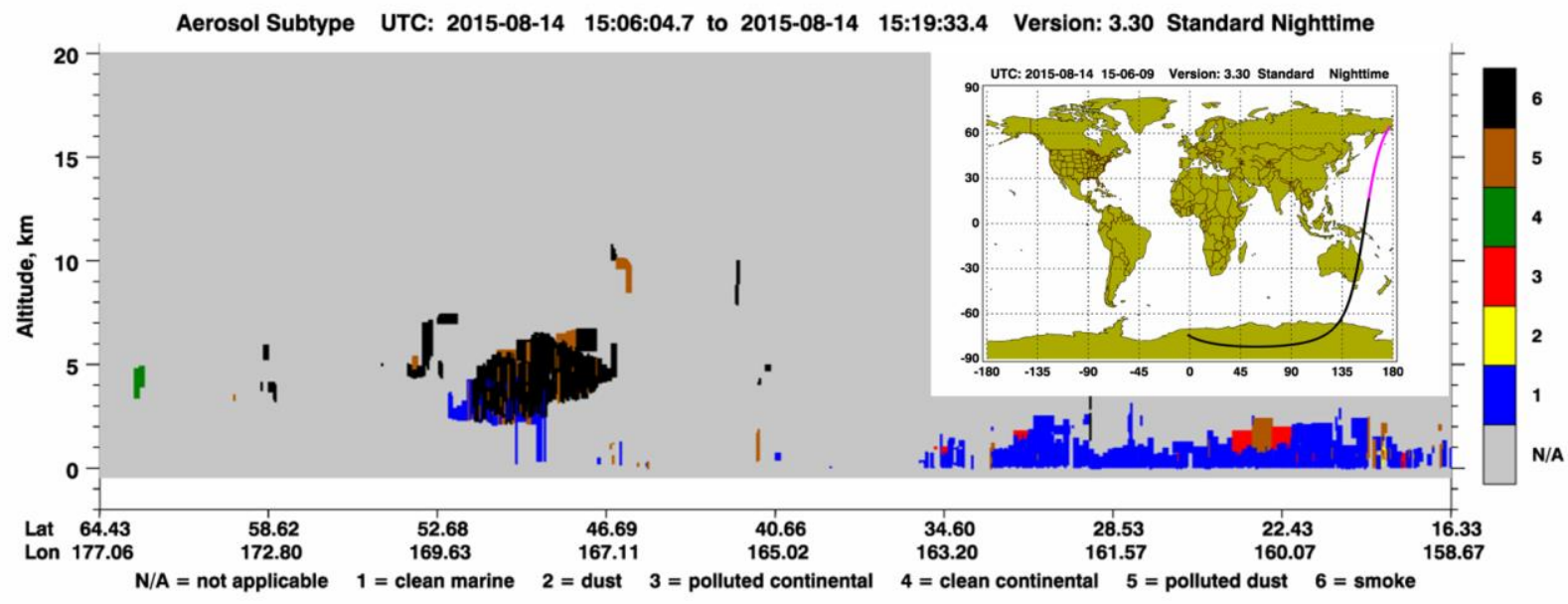

Figure S2. Similar plot as S2 for 8/14/2015 15:06 UTC confirming the eastward transport of a large Siberian smoke event. 


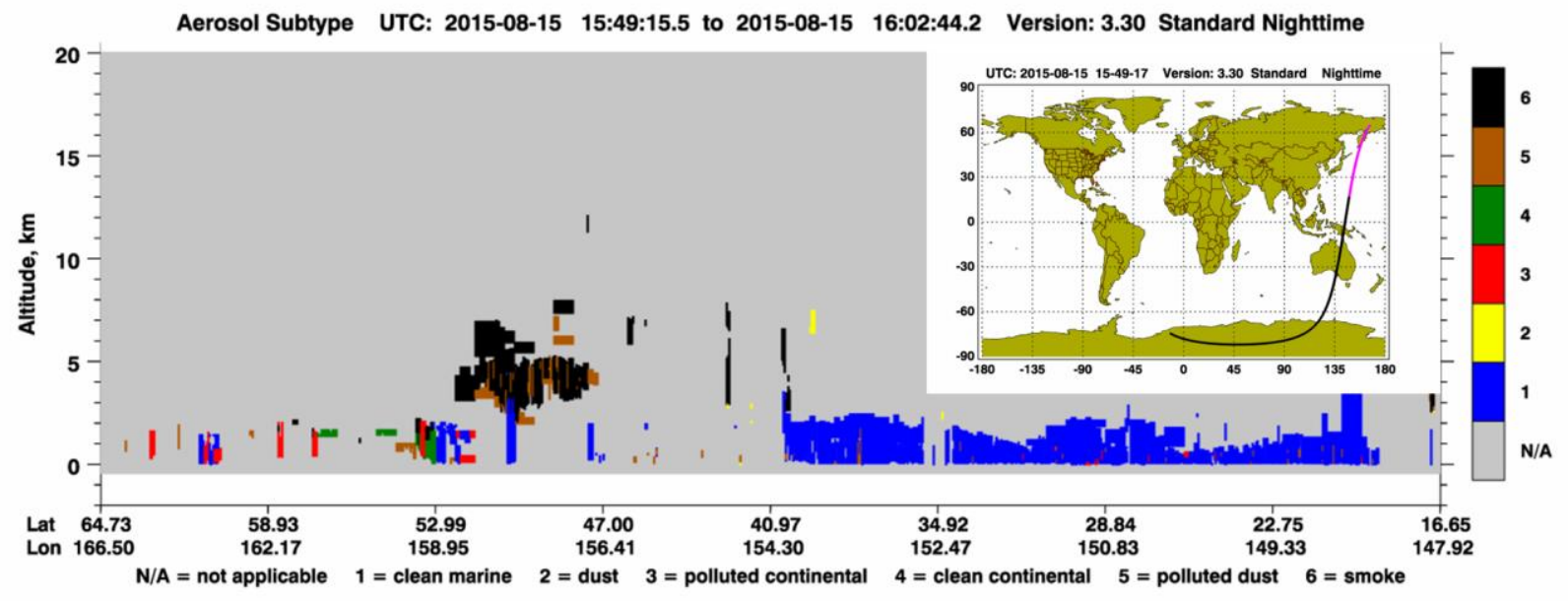

Figure S3. Similar plot as S2 for 8/15/2015 15:49 UTC. 


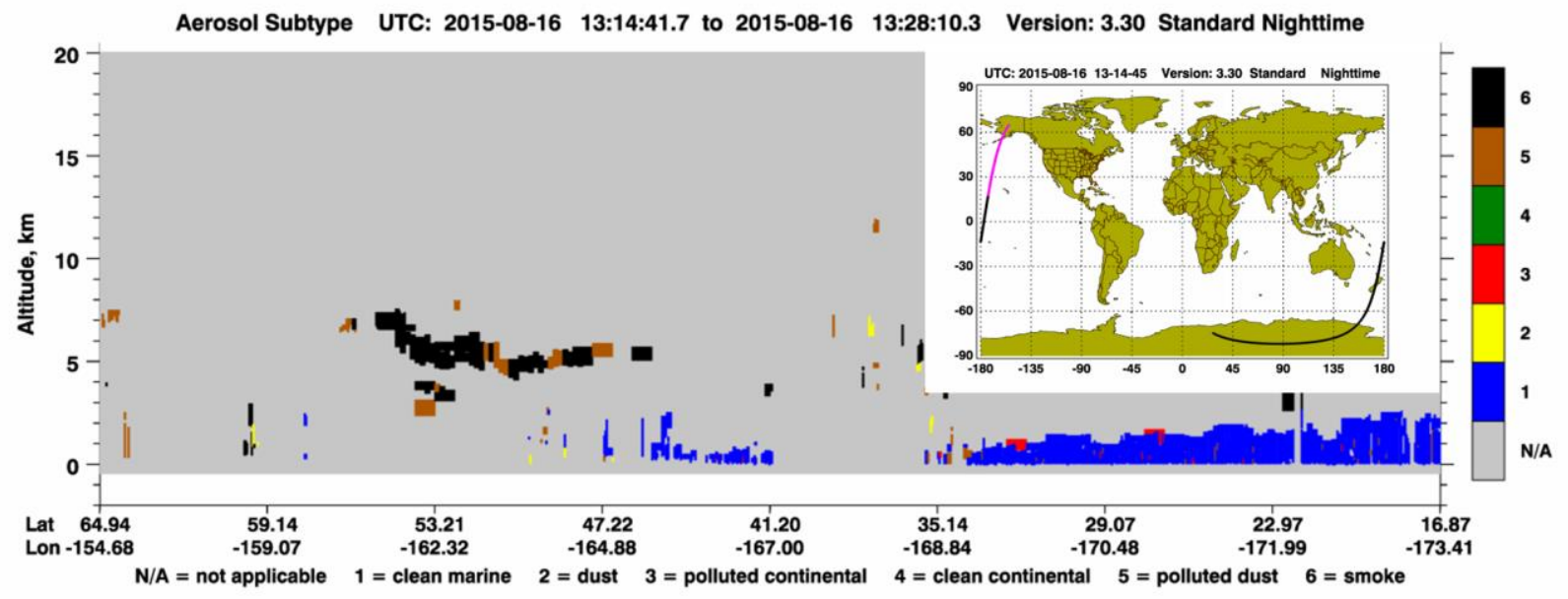

Figure S4. Similar plot as S2 for 8/16/2015 13:14 UTC. 